\title{
Emotional regulation and attachment style in previously untreated adolescents with attention deficit and hyperactivity disorder
}

\author{
Murat Eyuboglu' ${ }^{1}$, Damla Eyuboglu ${ }^{1 \oplus}$ \\ 'Eskisehir Osmangazi University, Faculty of Medicine, Department of Child and Adolescent Psychiatry, Eskisehir - Turkey
}

\begin{abstract}
Objective: The aim of this study was to evaluate difficulties with emotion regulation and attachment characteristics in adolescents diagnosed with attention deficit hyperactivity disorder (ADHD) for the first time and to compare these patients with healthy controls.

Method: This research was conducted as a cross-sectional study and included a healthy control group for comparison. A total of 48 untreated adolescents with ADHD and 51 healthy subjects participated in the study. A structural interview was used to determine psychiatric disorders. All of the participants were assessed using the Wechsler Intelligence Scale for ChildrenRevised. Emotion regulation and attachment characteristics were assessed with the Difficulties in Emotion Regulation Scale and the Experiences in Close Relationships Scale. The ADHD symptoms of the case group were evaluated using the Conners' Parent Rating Scale, which was completed by the patients' parents.

Results: Adolescents with ADHD displayed poorer performance in emotion regulation and had a higher avoidant attachment score. Furthermore, emotional regulation difficulties and attachment scores correlated with the severity of the ADHD.

Conclusion: Difficulty in emotion regulation and insecure-avoidant attachment styles were more common in untreated adolescents with ADHD. The study findings support the view that ADHD is a heterogeneous condition and that insecure attachment style and emotional regulation should be considered in the assessment and treatment of ADHD.
\end{abstract}

Keywords: Attention deficit hyperactivity disorder, attachment, emotional regulation

\section{INTRODUCTION}

Attention deficit hyperactivity disorder (ADHD) is one of the most common neurodevelopmental disorders of childhood and includes heterogeneous clinical symptoms, such as inattention, hyperactivity, and impulsivity (1). The core symptoms of ADHD are associated with significant impairment across the spectrum of social, cognitive, behavioral, and family functioning $(2,3)$. While executive functioning impairments have been central to cognitive theories of ADHD, recent studies have also suggested a focus on other psychological factors, such as emotional regulation and attachment.

Research has shown that these children have difficulty in social, emotional, and behavioral areas aside from the core symptoms of ADHD $(4,5)$. Young people with ADHD show greater emotional reactivity (6), higher levels of negative affect (7), and lower levels of emotional

How to cite this article: Eyuboglu M, Eyuboglu D. Emotional regulation and attachment style in previously untreated adolescents with attention deficit and hyperactivity disorder. Dusunen Adam The Journal of Psychiatry and Neurological Sciences 2020;33:228-236.

Correspondence: Murat Eyuboglu, Eskisehir Osmangazi University, Faculty of Medicine, Department of Child and Adolescent Psychiatry, 26480, Eskisehir - Turkey 
awareness than those without ADHD (8). Emotional regulation is the ability of individuals to evaluate and change their emotional reactions as needed. Impairments can lead to emotional dysregulation and weakened adaptive skills. Although emotion dysregulation is not among the cardinal symptoms in the current classification system, it was defined in the Diagnostic and Statistical Manual of Mental Disorders Third Edition (DSM-III) as a symptom associated with the disease (9). Despite not being included in the diagnostic criteria, emotional regulation is an important point that needs to be considered in the ADHD assessment process because of its effect on psychosocial, physical, and social areas (10). Epidemiological studies have also focused on emotional dysregulation. In a study of 5326 adolescents, $38 \%$ of children with ADHD were found to exhibit mood lability, which is 10 times greater than the presence determined in the normal population, including high rates in children with ADHD and no other comorbid psychiatric disorders (11). In another study conducted with 1500 children, emotional problems were demonstrated to have a greater impact on quality of life and self-esteem than hyperactivity and attention deficit (12). Children with ADHD and emotional regulation problems exhibit more impairment in peer relationships, family life, and academic performance than those with ADHD alone (4). In summary, emotional dysregulation is a clinical condition that can be common in children with ADHD and leads to adverse consequences.

There is a relationship between insecure attachment and $\mathrm{ADHD}$, and attachment problems may contribute to the development of ADHD (13-15). Some authors have stated that early attachment relationships may operate as organizers of the regulatory system, including the attentional system (16). Parent-child attachment may be a factor related to general psychopathology and ADHD symptoms, perhaps through its influence on the ability to self-regulate (17). Moreover, attachment style may have an effect on the clinical characteristics and course of ADHD and may be a precursor for future ADHD development (18). Children with ADHD typically have relationship problems with parents and they are more often exposed to childhood trauma and parental loss or separation $(19,20)$. Secure attachment is based on appropriate interaction between the parent and child, and the presence of inattention, hyperactivity, and impulsivity in the first years of life may prevent parents from receiving signals from their children and may lead to adverse effects on attachment development. Individuals with insecure attachment are more prone to developing behavioral and emotional regulation problems (21). It is also important to note that behavioral problems related to attachment pattern may also be influenced by other variables, such as characteristics of the particular child and environmental factors. Secure attachment has a positive effect on areas of competency that are often problematic for children with ADHD (13). A finding that $60 \%$ of children in the normal population were classified as having secure attachment and that fewer than $10 \%$ of children with ADHD displayed secure attachment supports a relationship between ADHD and insecure attachment $(22,23)$. In a review investigating the relationship between ADHD and attachment, emotional dysregulation was found to be an important characteristic of both ADHD and reactive attachment disorder (24). Although biological factors are more emphasized in the etiology of ADHD, attachment that begins in childhood and continues throughout life can be an important factor affecting the clinical course of ADHD, as in many psychiatric disorders. However, very few studies have examined attachment in relation to ADHD and the impact on the nature of the disorder.

Given this background, the objective of this study was to assess adolescents who had not been treated previously and were diagnosed with ADHD for the first time and then to compare them with healthy controls. The attachment characteristics of the adolescents with ADHD and the possible correlation of emotion dysregulation with ADHD symptoms were investigated. Based on the current literature, the following hypotheses were tested: (1) Adolescents with ADHD would display a more avoidant and anxious attachment style, (2) adolescents with ADHD would experience more emotional regulation difficulties compared with healthy adolescents, (3) attachment and emotional regulation scores would correlate with ADHD severity in the case group. Although there have been previous studies of various neuropsychological deficits, research examining the relationship between emotional regulation and attachment and ADHD is limited. The current study is offered as a contribution to the literature regarding the association between attachment pattern, emotional regulation, and ADHD.

\section{METHOD}

\section{Participants and Procedure}

This was a cross-sectional study with a healthy control group used for comparison. The subjects of the ADHD group were recruited at the Mardin Public Hospital Child and Adolescent Psychiatry outpatient clinic based on incidental sampling. The group included 48 
adolescents aged $12-17$ years $(14.7 \pm 1.7$ years $)$ and diagnosed with ADHD according to the DSM IV-TR criteria. The ADHD subjects were selected from adolescent patients who had never received any related medical or psychological treatment. A total of 69 subjects were initially selected to participate; however, 18 were excluded for at least 1 comorbid disorder other than oppositional defiant disorder (ODD) and 3 subjects were excluded for an estimated intelligence quotient (IQ) of $<80$. Due to the high comorbidity rate of ADHD and ODD, ODD was not defined as a criterion for exclusion. The inclusion criteria for the case group were (1) meet diagnostic criteria for at least 1 subtype of ADHD according to the DSM IV-TR, (2) demonstrate an IQ of $\geq 80$ on the Wechsler Intelligence Scale for Children (WISC-R), (3) no chronic medical illness requiring treatment. The exclusion criteria were (1) an estimated IQ $<80$ and (2) the presence of any of the following comorbidities: mental retardation, anxiety disorder, mood disorder, conduct disorder, psychotic disorders, and alcohol or other substance use. A total of 51 adolescents aged $12-17$ years ( $15.2 \pm 1.3$ years) who scheduled an appointment with the pediatrician and met the criteria, and who agreed to participate in the study along with their parents, were included in the study as the control group. Nine adolescents were excluded from the control group as a result of the determination of at least 1 psychiatric disorder.

All of the families and participants provided a signed informed consent form that was specifically designed for the current study. An interview of all of the children was conducted by 2 licensed child and adolescent psychiatrists using the Kiddie Schedule for Affective Disorders and Schizophrenia for School-Age Children-Present and Lifetime Version (K-SADS-PL) to determine the presence of any psychiatric disorders. The WISC-R intelligence test was applied to determine the intelligence level of all of the participants, in addition to the Difficulties in Emotion Regulation Scale (DERS) and the Experiences in Close Relationships Scale (ECRS). In addition, parents of the case group patients completed the Conners' Parent Rating Scale to assess ADHD severity. Ethical approval was obtained from Diyarbakir Gazi Yasargil Research and Training Hospital (Turkey) (Date: 17/07/2017, Number: 69).

\section{Measures}

Kiddie Schedule for Affective Disorders and Schizophrenia for School-Age Children-Present and Lifetime Version (K-SADS-PL): The K-SADS-PL is a semi-structured interview form developed to determine the previous and present psychopathologies of children and adolescents according to the DSM-III-R and DSMIV diagnostic criteria (25). A validity and reliability study of the Turkish version has been performed (26).

Conners' Parent Rating Scale (CPRS-48): The CPRS is used as a review instrument to determine behavioral problems observed in children and as an additional criterion for the diagnosis, as well as for monitoring and grading treatment (27). The CPRS short form includes additional items for psychosomatic problems and anxiety, in addition to items related to hyperactivity and learning and behavioral problems. The validity and reliability studies of the Turkish version yielded a Cronbach's alpha value of 0.90 (28).

Experiences in Close Relationships Scale (ECRS): This scale, developed by Brennan and Shaver, measures anxiety and avoidance, which are 2 main dimensions of attachment (29). The scale consists of 36 items, and each dimension is measured with 18 items. Participants assess each item using a 7-point Likert-type scale ( $1=$ does not describe me at all, $7=$ fully describes me). The Turkish version of the scale has been validated (30). The Cronbach's alpha value of the avoidant and anxious domains were determined to be 0.90 and 0.94 , respectively (30).

Difficulties in Emotion Regulation Scale (DERS): The scale developed by Gratz and Roemer (31) consists of the subdimensions of awareness, clarity, non-accept, strategies, impulse, and goals, and contains 36 items. In completing this self-report scale, adolescents are asked to read the items and to mark the closest expression based on the frequency that applies ( $1=$ almost never, $5=$ almost always). Although a cut-off score has not been determined for the scale, high scores point to the presence of more severe difficulties in emotional regulation. A validity and reliability study of the Turkish scale was conducted and yielded a Cronbach's alpha value of 0.94 (32).

\section{Statistical Analysis}

Data analyses were performed using IBM SPSS Statistics for Windows, Version 22.0 software (IBM Corp., Armonk, NY, USA). The measured variables were presented as the mean $\pm \mathrm{SD}$ and the categorical variables as percentage and number. The Kolmogorov-Smirnov test and histograms were used to evaluate the distribution of the numeric variables. Student's t-test was used to compare numerical variables with normal distribution, and the Mann-Whitney $U$ test, a nonparametric test, was used for those with a nonnormal distribution. The categorical variables were 
assessed with Pearson's chi-squared test and Fisher's exact test. To determine the direction and the level of correlation between the numerical variables, Pearson's correlation test was used for those exhibiting a normal distribution and Spearman's correlation test for the nonnormally distributed numerical variables. A multivariate analysis of covariance (MANCOVA) was used to identify effect of covariates such as age and sex. The Wilk's lambda test results are reported in the results section. Logistic regression analyses were conducted to assess the possible effect of ADHD on independent variables. Odds ratios and $95 \%$ confidence intervals (95\% CIs) were computed. The value of statistical significance was $\mathrm{p}<0.05$.

\section{RESULTS}

The mean age of the case group was $14.7 \pm 1.7$ years and that of the control group was $15.2 \pm 1.3$ years. In terms of gender, $85.4 \%(\mathrm{n}=41)$ of the case group was male and $14.6 \%(\mathrm{n}=7)$ was female. In the control group, $52.9 \%$ $(\mathrm{n}=27)$ were males and $47.1 \%(\mathrm{n}=24)$ were females. As expected, given the higher prevalence of ADHD in males, there were more males in the case group than in the control group. A significant difference was found between the 2 groups in terms of gender (chi-squared test: $\left.\chi^{2}=12.1, \mathrm{df}=1 ; \mathrm{p}<0.001\right)$. The academic achievement of the adolescents with ADHD was significantly lower than that of the control group (chi-squared test: $\chi^{2}=37$, $\mathrm{df}=4 ; \mathrm{p}<0.001)$.

Among the ADHD group, 35.4\% $(n=17)$ were smokers, while $13.7 \%$ of the control group were smokers, which represented a statistically significant difference (chi-squared test: $\chi^{2}=6.3, \mathrm{df}=1 ; \mathrm{p}=0.012$ ). Five adolescents in the case group did not attend school, compared to 1 member of the control group. No significant differences were observed between the groups in terms of sociodemographic characteristics, such as age, number of siblings, parental status, parental age, and family income ( $\mathrm{p}>0.05)$.

Among the adolescents in the case group, $81.3 \%$ $(n=39)$ had mixed-type ADHD, 14.6\% $(n=7)$ had predominant attention deficit-type ADHD, and 4.2\% $(n=2)$ had predominant hyperactivity-type ADHD. Additionally, 8 of the adolescents (16.6\%) in the case group had an ODD comorbidity. The mean CPRS score of the case group was $52.2 \pm 17.7$.

The total WISC-R score of the case group was $95 \pm 4$ and that of the control group was $97 \pm 2$. No significant difference was found between the groups in terms of intelligence level $(\mathrm{p}>0.05)$.
The case and the control groups were compared using the Mann-Whitney $U$ test because the ECRS subscale and total scores did not exhibit a normal distribution. Higher ECRS scores indicate a more disturbed attachment pattern. The ECRS avoidance and total attachment scores of the ADHD adolescents were higher than those of the control group (Table 1). No significant difference was found between the 2 groups in terms of the anxiety scores.

The DERS subscale scores were also compared with the Mann-Whitney $U$ test as a result of non-normal distribution. Subscales related to awareness, clarity, impulse, and goals, as well as the total DERS scores of the case group were significantly higher. Higher DERS scores reflect difficulty in emotional regulation. No difference was found between the 2 groups in the nonacceptance and strategies subscale scores (Table 1).

\section{Correlations}

In the present study, the correlation between the total score of the CPRS and the emotional dysregulation and attachment scores of the case group was examined to investigate the effect of the ADHD symptoms on attachment and emotional regulation. A significant correlation was found between the total CPRS score, the total DERS score, and the avoidant attachment score (Table 2). Additionally, there was a significant correlation between the insecure attachment and emotional regulation scores $(r h o=0.614 ; \mathrm{p}<0.001)$.

\section{Analysis of Covariance}

MANCOVA was used to investigate the effect of age and gender in group comparisons. Attachment and emotional regulation scores were selected as dependent variables, and sex and age were selected as covariates. It was determined that the covariates of age (Wilks' lambda $=0.974, \mathrm{~F}=740, \mathrm{p}=0.53$, partial $\eta^{2}=0.02$ ) and sex (Wilks' lambda $=0.987, \mathrm{~F}=0.376, \mathrm{p}=0.78$, partial $\eta^{2}=0.01$ ) had no effect in group differences. After controlling for sex and age, there were still significant differences in the insecure attachment and emotion regulation scores between the case and control groups (Table 3).

\section{Logistic Regression Analysis}

Binary logistic regression analysis was used to assess the effect of ADHD on measured variables. A diagnosis of ADHD was added as a dependent variable. Attachment scores, emotional regulation scores, and sociodemographic characteristics that were significant in the previous analyses were added to the model as independent variables. The results indicated that a 
Table 1: Comparison of the case and control group ECRS and DERS scores

\begin{tabular}{|c|c|c|c|c|c|}
\hline & \multicolumn{2}{|c|}{ Case group } & \multicolumn{2}{|c|}{ Control group } & \multirow[b]{2}{*}{$\mathbf{p}^{*}$} \\
\hline & $M$ & SD & M & SD & \\
\hline Anxious attachment score & 66.2 & 20.5 & 62.5 & 21.5 & 0.234 \\
\hline Avoidant attachment score & 67.6 & 16.4 & 53.9 & 12.5 & $<0.001$ \\
\hline Total ECRS score & 133.8 & 27.4 & 116.5 & 23.2 & 0.004 \\
\hline Awareness & 15.3 & 4.7 & 12.4 & 3.0 & 0.001 \\
\hline Clarity & 13.3 & 4.2 & 10.6 & 3.5 & 0.001 \\
\hline Non-accept & 15 & 6.5 & 13.8 & 5.6 & 0.480 \\
\hline Strategies & 21.8 & 7.8 & 19.1 & 6.0 & 0.072 \\
\hline Impulse & 18.8 & 7.1 & 13.4 & 5.8 & $<0.001$ \\
\hline Goals & 18 & 5 & 15.7 & 4.5 & 0.020 \\
\hline Total DERS score & 102.2 & 24.5 & 85 & 20.0 & $<0.001$ \\
\hline
\end{tabular}

*Mann-Whitney U test. DERS: Difficulties in Emotion Regulation Scale, ECRS: Experiences in Close Relationships Scale, SD: Standadr deviation, M: Mean

Table 2: The relationship between the ADHD severity and emotional dysregulation and attachment in the case group

\begin{tabular}{lccccccc} 
& \multicolumn{3}{c}{ DERS } & \multicolumn{4}{c}{ ECRS } \\
\cline { 2 - 8 } & & & & & \multicolumn{2}{c}{ Avoidant attachment } & \multicolumn{2}{c}{ Anxious attachment } \\
\cline { 3 - 8 } & & rho & p & rho & p & rho & p \\
\hline Conners' Parent Rating Scale score & 0.481 & 0.001 & & 0.361 & 0.036 & 0.238 & 0.16 \\
\hline
\end{tabular}

Spearman's correlation analysis. ADHD: Attention deficit hyperactivity disorder; ECRS: Experiences in Close Relationships Scale; DERS: Difficulties in Emotion Regulation Scale

Table 3: Results of MANCOVA for attachment and emotional regulation in the case and control groups after controlling for age and sex

\begin{tabular}{|c|c|c|c|c|c|}
\hline & Type III sum of squares & df & $\mathbf{F}$ & $\mathbf{p}$ & Partial $\eta^{2}$ \\
\hline ECRS total score & 6849.0 & 3 & 3.6 & 0.018 & 0.11 \\
\hline Avoidant attachment & 4477.2 & 3 & 7.2 & $<0.001$ & 0.20 \\
\hline Anxious attachment & 305.0 & 3 & 0.2 & 0.88 & 0.008 \\
\hline DERS total score & 7601.6 & 3 & 5.4 & 0.002 & 0.16 \\
\hline Awareness & 251.7 & 3 & 6.0 & 0.001 & 0.17 \\
\hline Clarity & 285.1 & 3 & 7.4 & $<0.001$ & 0.20 \\
\hline Non-accept & 74.9 & 3 & 0.7 & 0.57 & 0.02 \\
\hline Strategies & 188.8 & 3 & 1.3 & 0.26 & 0.05 \\
\hline Impulse & 837.8 & 3 & 6.8 & $<0.001$ & 0.19 \\
\hline Goals & 249.2 & 3 & 3.9 & 0.012 & 0.12 \\
\hline
\end{tabular}

DERS: Difficulties in Emotion Regulation Scale; ECRS: Experiences in Close Relationships Scale; MANCOVA: Multivariate analysis of covariance

diagnosis of ADHD was significantly related to an increased risk of emotion dysregulation, insecure attachment, and smoking (Table 4).

\section{DISCUSSION}

In this study, compared with healthy adolescents, untreated adolescents with ADHD were found to have poorer emotional regulation skills and greater insecureavoidant attachment characteristics. Moreover, a greater severity of ADHD was associated with more emotional dysregulation and attachment difficulty. Emotional regulation problems and attachment characteristics appear to have a great effect on functioning and are important points that should be considered in the evaluation of ADHD. These 2 conditions may also be factors that increase morbidity in children with untreated ADHD.

We found a significant difference in the awareness, clarity, impulse, and goals subscales and the total scores 
Table 4: Final model of logistic regression analyses

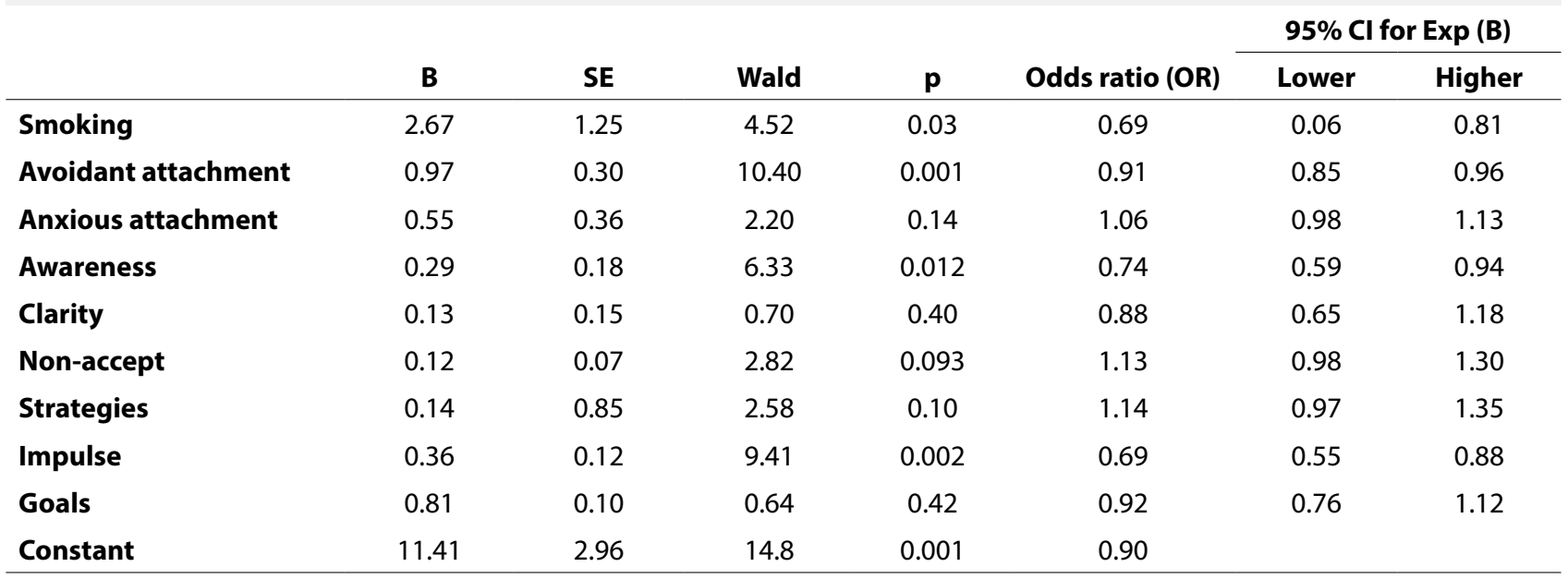

$B$ is the unstandardized B regression coefficient; SE is the standard error of the regression coefficient; Wald is the chi-squared test value; Sig. is the $p$ value of the Wald chi-squared test; odds ratio is the exponential of the regression coefficient

of the DERS. The awareness subscale reflects the lack of awareness of emotional reactions, clarity indicates the insufficient understanding of emotional reactions, impulse conveys difficulties in controlling impulses while experiencing negative emotions, and goals expresses difficulties in focusing while experiencing negative emotions (32). As observed from our findings using this scale, the adolescents with untreated ADHD were found to have difficulty with understanding emotional situations, impulse control, and behavior regulation areas when compared with their healthy peers in emotional situations. According to Barkley's model, compared with those without ADHD, the inhibitory deficit in ADHD individuals leads to more emotional reactivity or emotional dysregulation in emotional situations $(33,34)$. Similarly, children and adolescents with ADHD have been found to be emotionally more reactive than controls (6). Consistent with previous studies, we also recorded the presence of emotional dysregulation and emotional reactivity in these children. Moreover, the fact that these findings correlated with ADHD severity reveals how great the effect of ADHD is on children's lives. The exclusion of comorbid psychiatric conditions, primarily conduct disorder, suggests that emotional regulation difficulties can be more specific to ADHD. Emotional dysregulation is not unique to ADHD. However, the addition of this condition to ADHD increases the impairment in functioning (4), and the demonstration of the improvement effect of ADHD treatment on emotional regulation increases the importance of early treatment in ADHD. In support of this finding, a study conducted of 79 ADHD children with emotional dysregulation showed that comorbidity, social deficiency, and continuity of ADHD symptoms were greater after 4 years in these children than in those with ADHD without emotional dysregulation (35). A similar study showed that people with ADHD and emotional symptoms have a significantly lower quality of life and poorer social adjustment compared with individuals with ADHD but no emotional symptoms (36). Many studies have focused on psychiatric comorbidities, such as substance use disorder, in the adolescence period of children with untreated ADHD. Emotional problems, which are known to have a great effect on quality of life and self-respect, have remained in the background. Moreover, in adolescence, which is an important period for the development of identity, children with ADHD are at risk of being excluded by their peers or being exposed to bullying because of behaviors associated with emotional regulation. From the etiological perspective, neurophysiological changes have been demonstrated in emotional dysregulation and in ADHD. Such abnormalities in children with ADHD, especially abnormalities in parasympathetic mechanisms, including emotional regulation (37), shows that the emotion dysregulation seen in ADHD is also associated with neurochemical changes in brain. However, emotional regulation in ADHD is still not fully understood. Neuropsychological tests related to emotional control (impulsivity, self-regulation, and executive functioning in positive and negative emotions) have also shown that the processing of emotional stimulation is impaired in ADHD (38).

The findings of this study indicated that adolescents with ADHD had more avoidant attachment characteristics. This insecure form of attachment can develop when the child's signals remain unanswered or 
are misinterpreted as a result of inappropriate behaviors or the late responses of the attachment figure. Children with insecure-avoidant attachment may not be fully capable of predicting the behavior of individuals compared with those who have secure attachment. Furthermore, children with secure attachment have better social competence, emotional regulation, and psychosocial well-being than children with insecure attachment, who are anxious, worried, or disorganized $(39,40)$. Moreover, insecure attachment has been reported to be a risk factor that increases socioemotional and behavioral maladjustment in adolescents with ADHD (41). Attachment affects the development of self-regulation skills. The finding of the present study suggests that in addition to their difficulties in emotion regulation, children with ADHD may have difficulty handling close relationships in the early period of life. Consequently, the quality of attachment that is expected to develop in a healthy way in early childhood can also be adversely affected. The fact that the attachment characteristics of children with ADHD are affected by the severity of the disorder also suggests a relationship between insecure-avoidant attachment and ADHD. The opposite can also be considered: ADHD severity may increase according to the degree of attachment impairment. Previous studies have reported that this condition was associated with externalizing and behavioral problems in individuals with insecure attachment $(42,43)$. In our study, the exclusion of disorders other than ODD that could lead to externalizing problems increases the probability of a relationship between attachment and ADHD. However, it should be kept in mind that ODD, which is a common comorbid condition with ADHD, may also be associated with attachment and emotional regulation. Due to the nature of this disorder, the parent-child relationship can be affected at an early age of life. Yet, an important point that should be noted is that defining the cause-andeffect relationship between the attachment pattern, emotional regulation, and ADHD remains difficult. The relationship between these characteristics is probably bi-directional, and the presence of one poses a risk for another.

The limitations of the study should be considered during the assessment of the study findings. First of all, the present study was limited to a cross-sectional analysis, and causal effects need to be validated in future prospective studies. Self-report questionnaires were used. In particular, parental ratings which have been found to be less reliable than teacher ratings for externalizing behavior problems, were used to assess externalizing behaviors (44). This also introduced the risk that there could be bias in the data collected. Although comorbid conditions were excluded from the study, ODD was not eliminated. Children with ODD are known to have externalizing problems. The number of ODD samples may decrease the power of our findings, which then could be insufficient to make a generalization. The sample size also made it difficult to have the power to test the diagnosis according to ADHD subgroups. It would be also interesting to examine the effect of comorbid conditions on attachment and emotional regulation. Parental psychopathology was based on their own responses and we did not conduct clinical interviews with the parents to detect possible disorders, such as ADHD or other psychiatric conditions. Parental psychopathology likely has an influence on parent-child attachment. Future research using a randomized design with a larger sample size and using multiple informant reports with a structured interview would be valuable.

In conclusion, although the relationship of emotional regulation and attachment to ADHD is not yet fully understood, difficulties in emotional regulation and insecure-avoidant attachment style have been determined to be more common in untreated ADHD adolescents and to affect the clinical course. While several factors may have an impact on emotional regulation and attachment, MANCOVA suggested that our findings demonstrate a significant relationship with ADHD, regardless of age and gender. Emotional dysregulation, especially in response to negative situation and emotions, can lead to significant problems in the social relations of these individuals. Our findings are also important in terms of demonstrating the effect of attachment style on the life of adolescents. Secure attachment is protective in the prolongation of ADHD symptoms to the adulthood period, and the positive effect of ADHD treatment, especially on emotional regulation, is important in assessing the findings of the present study. Due to their symptoms, children with ADHD may be at risk of impairment of the motherinfant interaction. Therefore, this should absolutely be kept in mind by clinicians who evaluate childhood ADHD symptoms. Furthermore, developing appropriate behavioral intervention programs for this condition that could affect the attachment style may be an option. The relationship between emotional regulation and ADHD has the capacity to affect almost every aspect of life. In consideration of all of these, the effect of the lack of early intervention and treatment on the lives of children and adolescents with ADHD 
appears to be greater than expected. The treatment of this disorder will not only reduce the severity of the illness, but will also promote improvement in many areas, particularly the quality of life, peer relations, and social life of the patient.

\begin{tabular}{|c|c|c|}
\hline \multicolumn{2}{|c|}{ Contribution Categories } & \multirow{2}{*}{$\begin{array}{l}\text { Author Initials } \\
\text { M.E., D.E. }\end{array}$} \\
\hline \multirow{3}{*}{ Category 1} & Concept/Design & \\
\hline & Data acquisition & M.E., D.E. \\
\hline & Data analysis/Interpretation & M.E. \\
\hline \multirow{2}{*}{ Category 2} & Drafting manuscript & M.E. \\
\hline & Critical revision of manuscript & M.E., D.E. \\
\hline Category 3 & Final approval and accountability & M.E., D.E. \\
\hline \multirow{2}{*}{ Other } & Technical or material support & N/A \\
\hline & Supervision & N/A \\
\hline
\end{tabular}

Ethics Committee Approval: Ethical approval was obtained from Diyarbakir Gazi Yasargil Research and Training Hospital (Turkey) (Date: 17/07/2017, Number: 69).

Informed Consent: Written informed consent obtained.

Peer-review: Externally peer-reviewed.

Conflict of Interest: The authors declare that there is no conflict of interest.

Financial Disclosure: This research did not receive any specific grant from funding agencies in the public, commercial, or not-forprofit sectors.

\section{REFERENCES}

1. American Psychiatric Association. Diagnostic and Statistical Manual of Mental Disorders Fifth Ed. (DSM-5).Washington DC: American Psychiatric Publishing, 2013.

2. Loe IM, Feldman HM. Academic and educational outcomes of children with ADHD. Ambul Pediatr 2007; 7:82-90.

3. Mash EJ, Barkley RA (editors). Child Psychopathology. Second ed., New York: Guilford Press, 2003.

4. Wehmeier PM, Schacht A, Barkley RA. Social and emotional impairment in children and adolescents with ADHD and the impact on quality of life. J Adolesc Health 2010; 46:209-217.

5. McQuade JD, Hoza B. Peer problems in Attention Deficit Hyperactivity Disorder: current status and future directions. Dev Disabil Res Rev 2008; 14:320-324.

6. Jensen SA, Rosén LA. Emotional reactivity in children with attention-deficit/hyperactivity disorder. J Atten Disord 2004; 8:53-61.

7. Braaten EB, Rosén LA. Self-regulation of affect in attention deficit-hyperactivity disorder (ADHD) and non-ADHD boys: differences in empathic responding. J Consult Clin Psychol 2000; 68:313-321.

8. Factor PI, Rosen PJ, Reyes RA. The Relation of Poor Emotional Awareness and Externalizing Behavior Among Children With ADHD. J Atten Disord 2016; 20:168-177.
9. American Psychiatric Association. Diagnostic and Statistical Manual of Mental Disorders Third ed., Washington DC: American Psychiatric Publishing, 1980.

10. Gross JJ. Emotion regulation: Affective, cognitive, and social consequences. Psychophysiology 2002;39:281-291.

11. Stringaris A, Goodman R. Mood lability and psychopathology in youth. Psychol Med 2009; 39:1237-1245.

12. Riley AW, Spiel G, Coghill D, Döpfner M, Falissard B, Lorenzo MJ, et al; ADORE Study Group. Factors related to health-related quality of life (HRQoL) among children with ADHD in Europe at entry into treatment. Eur Child Adolesc Psychiatry. 2006 Dec;15(Suppl.1):I38-45.

13. Clarke L, Ungerer J, Chahoud K, Johnson S, Stiefel I. Attention deficit hyperactivity disorder is associated with attachment insecurity. Clin Child Psychol Psychiatry 2002; 7:179-198.

14. Pinto C, Turton P, Hughes P, White S, Gillberg C. ADHD and infant disorganized attachment: a prospective study of children next-born after stillbirth. J Atten Disord 2006; 10:83-91.

15. Green J, Stanley C, Peters S. Disorganized attachment representation and atypical parenting in young school age children with externalizing disorder. Attach Hum Dev 2007; 9:207-222.

16. Fearon RM, Belsky J. Attachment and attention: protection in relation to gender and cumulative social-contextual adversity. Child Dev 2004; 75:1677-1693.

17. Fonagy P, Target M. Early intervention and the development of self regulation. Psychoanal Inq 2002; 22:307-335.

18. Franc N, Maury M, Purper-Ouakil D. ADHD and attachment processes: are they related?. Encephale 2009; 35:256-261. (French)

19. Eng W, Heimberg RG, Hart TA, Schneier FR, Liebowitz MR. Attachment in individuals with social anxiety disorder: the relationship among adult attachment styles, social anxiety, and depression. Emotion 2001; 1:365-380.

20. Sroufe LA. Psychopathology as an outcome of development. Dev Psychopathol 1997; 9:251-268.

21. Cassidy J. Emotion regulation: influences of attachment relationships. Monogr Soc Res Child Dev 1994; 59:228-249.

22. Shmueli-Goetz Y, Target M, Fonagy P, Datta A. The Child Attachment Interview: a psychometric study of reliability and discriminant validity. Dev Psychol 2008; 44:939-956.

23. Storebø OJ, Gluud C, Winkel P, Simonsen E. Social-skills and parental training plus standard treatment versus standard treatment for children with ADHD--the randomised SOSTRA trial. PLoS One 2012; 7:e37280.

24. Fonagy P, Gergely G, Jurist EL, Target M. Affect Regulation, mentalization, and the development of the self. New York NY: Other Press, 2002.

25. Kaufman J, Birmaher B, Brent D, Rao U, Flynn C, Moreci P, et al. Schedule for Affective Disorders and Schizophrenia for SchoolAge Children-Present and Lifetime Version (K-SADS-PL): initial reliability and validity data. J Am Acad Child Adolesc Psychiatry 1997; 36:980-988. 
26. Gokler B, Unal F, Pehlivanturk B, Kultur EC, Akdemir D, Taner Y. Reliability and validity of schedule for affective disorders and schizophrenia for school age children - Present and lifetime version - Turkish version (K-SADS-PL-T). Cocuk ve Genclik Ruh Sagligi Dergisi 2004; 11:109-116. (Turkish)

27. Conners CK. A teacher rating scale for use in drug studies with children. Am J Psychiatry 1969; 126:884-888.

28. Dereboy C, Senol S, Sener S, Dereboy F. Validation of the Turkish versions of the short-form conners' teacher and parent rating scales. Turk Psikiyatri Derg 2007; 18:48-58. (Turkish)

29. Brennan KA, Clark CL, Shaver PR. Self-report measurement of adult attachment: an integrative overview: In Simpson JA, Rholes WS (editors). Attachment Theory and Close Relationships. New York: Guilford Press, 1998, 46-76.

30. Sumer N. Categorical and dimensional comparison of the adult attachment measures. Turkish Journal of Psychology 2006; 21:124.

31. Gratz KL, Roemer L. Multidimensional assessment of emotion regulation and dysregulation: Development, factor structure, and initial validation of the Difficulties in Emotion Regulation Scale. J Psychopathology Behav 2004: 26;41-54.

32. Ruganci RN. The Relationship among attachment style, affect regulation, psychological distress and mental construction of the relational world. $\mathrm{PhD}$ Thesis, Middle East Technical University, Department of Psychology, Ankara, 2008.

33. Barkley RA. Attention-deficit/hyperactivity disorder, selfregulation, and time: toward a more comprehensive theory. J Dev Behav Pediatr 1997; 18:271-279.

34. Barkley RA. Behavioral inhibition, sustained attention, and executive functions: constructing a unifying theory of ADHD. Psychol Bull 1997; 121:65-94.
35. Shaw P, Stringaris A, Nigg J, Leibenluft E. Emotion dysregulation in attention deficit hyperactivity disorder. Am J Psychiatry 2014; 171:276-293.

36. Surman CB, Biederman J, Spencer T, Miller CA, McDermott KM, Faraone SV. Understanding deficient emotional self-regulation in adults with attention deficit hyperactivity disorder: a controlled study. Atten Defic Hyperact Disord 2013; 5:273-281.

37. Musser ED, Backs RW, Schmitt CF, Ablow JC, Measelle JR, Nigg JT. Emotion regulation via the autonomic nervous system in children with attention-deficit/hyperactivity disorder (ADHD). J Abnorm Child Psychol 2011; 39:841-852.

38. van Stralen J. Emotional dysregulation in children with attentiondeficit/hyperactivity disorder. Atten Defic Hyperact Disord 2016; 8:175-187.

39. Grossmann KE, Grossmann K, Waters E (editors). Attachment from infancy to adulthood. New York, NY: Guilford Press 2006.

40. Kerns KA, Richardson RA (editors). Attachment in middle school. New York NY: Guilford Press, 2005.

41. Thorell LB, Rydell AM, Bohlin G. Parent-child attachment and executive functioning in relation to ADHD symptoms in middle childhood. Attach Hum Dev 2012; 14:517-532.

42. DeVito C, Hopkins J. Attachment, parenting, and marital dissatisfaction as predictors of disruptive behavior in preschoolers. Dev Psychopathol 2001; 13:215-231.

43. Greenberg MT, Speltz ML, DeKlyen M, Jones K. Correlates of clinic referral for early conduct problems: variable- and personoriented approaches. Dev Psychopathol 2001; 13:255-276.

44. Oosterlaan J, Scheres A, Sergeant JA. Which executive functioning deficits are associated with $\mathrm{AD} / \mathrm{HD}, \mathrm{ODD} / \mathrm{CD}$ and comorbid AD/HD+ODD/CD? J Abnorm Child Psychol 2005; 33:69-85. 